\title{
Improving the reliability of temperature measurements taken with clinical infrared ear thermometers
}

\section{- Design and establishment of a new calibration system traceable to the national standards}

\author{
Juntaro Ishii \\ [Translation from Synthesiology, Vol.1, No.1, p.47-58 (2008)]
}

\begin{abstract}
Infrared ear thermometer was developed and rapidly spread in Japan in the late 1990's, but users began to question the reliability of the temperature readings. AIST developed a new national standard for the calibration and conformity assessment of new ear thermometers, and also designed and organized the traceability system, conducted technological verification, and improved reliability of measured temperature, to meet the demands of Japanese industry and consumers. An international comparison of national measurement standards among Germany, UK, and AIST was conducted and the equality of the standards was experimentally verified to maintain international reliability.
\end{abstract}

Keywords: Standard, traceability, infrared, clinical thermometer, reliability, blackbody, radiance temperature

\section{Introduction}

Along with blood pressure and heart rate, body temperature is one of the most basic vital signs of the human body that is used for medical diagnosis and health management. Since body temperature measurements are taken at home as well as in medical institutions by physicians and nurses, clinical thermometers require high reliability and utility as measuring instrument.

Mercury-in-glass thermometers have been used for a long time, but there were problems of glass breakage and use of mercury that is harmful to the human body. Later, when high-precision thermistor was developed as temperature sensor, clinical electronic thermometers with thermistor sensors became commercially available, and its use spread rapidly due to ease of handling and safety. It is widely used to this day. However, there were still problems with clinical electronic thermometers since the sensor must be held close to measured body-site such as the armpit for around five minutes to measure the body temperature accurately making it unpractical for emergency patients in serious condition, and temperature measurement in newborns and infants were difficult.
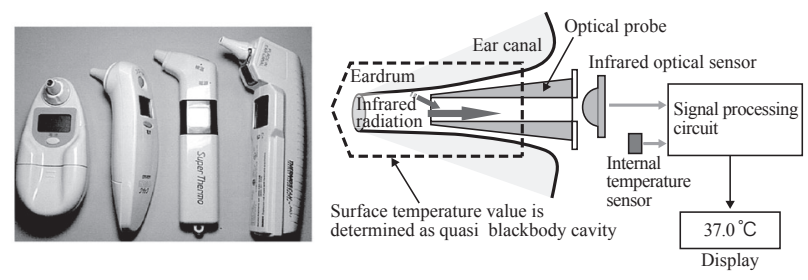

Fig. 1 External appearance and structure of ear thermometer
In the 1990s, a U.S. company developed a new infrared clinical thermometer in which measurement was taken by inserting the sensor probe into the ear canal (hereinafter will be called "ear thermometer"), and was marketed in the United States and Europe. Figure 1 shows the external appearance of ear thermometers and the schematic diagram of the measurement principle. Ear thermometer consists of optical probe, infrared sensor, compensating internal temperature sensor, signal processing circuit, and display unit. The tip of the optical probe is inserted into the ear canal, the intensity (radiance ${ }^{\text {Term } 1}$ ) of infrared radiation at around $10 \mu \mathrm{m}$ wavelength emitted from eardrum and skin surface of inner ear canal is measured, and the temperature of measured part is determined from the relation of Planck's law of thermal radiation ${ }^{\text {Term } 2}$. The calibration of temperature reading is conducted by using the ear thermometer to measure the radiation from ideal blackbody cavity for which accurate temperature is known.

The human skin surface has emissivity ${ }^{\text {Term } 3}$ close to unity in the thermal infrared wavelength region, so it is a suitable object for infrared radiation thermometry. The method of measuring skin surface temperature from thermal radiation measurement has been also applied successfully to breast cancer diagnosis technology using thermograph. In newly developed ear thermometers, eardrum and surrounding ear canal were selected as cavity to be measured, and therefore effective emissivity $(\varepsilon)$ of the measured area approached the condition of almost ideal blackbody $(\varepsilon=1)$, and the accuracy of body temperature measurement was greatly improved. Also, measurement could be accomplished in a short time of about 1 second by introducing advanced infrared sensor technology. This ear thermometers overcame the issues of conventional contact type (thermal equilibrium type) clinical thermometer, and drew attention as third-generation clinical

Metrology Institute of Japan, AIST Tsukuba Central 3, Tsukuba, Ibaraki 305-8563, Japan E-mail : j-ishii@aist.go.jp 
thermometer that enabled "short measurement time" and "non-contact measurement" "[1].

In Japan, ear thermometers were introduced as instrument for medical specialists in the beginning of the 1990s. In 1996, it was formally approved as a medical device for general use, and became commercially available. The number of production and sales of ear thermometers in the Japanese market increased rapidly, and in a few years, reached around one million a year. The reasons for this rapid expansion were the fact that new ear thermometer satisfied user demands, and that the manufacturers had high hopes for this new innovative product that appeared in clinical thermometer market that reached maturity since the development of clinical electronic thermometer about a quarter of a century $\operatorname{ago}^{[2]}$.

On the other hand, with rapid diffusion of ear thermometers, questions and claims against the reliability of temperature readings of ear thermometers were made by users and consumer organizations, and drew attention of mass media such as newspapers and magazines. Therefore, as the national metrology institute of Japan, AIST started a research project to improve measurement accuracy of ear thermometers. After adjustments with manufacturers, users, and administrative bodies, the traceability system was established by 2002 , and technological and social infrastructures were laid to maintain accuracy of temperature readings of ear thermometer.

Also, the standard facility for calibration of ear thermometer which resulted from this research was provided to national standard institutions of Asian countries when severe acute respiratory syndrome (SARS) epidemic occurred in Asia in 2003 , and helped to prevent the spread of infection ${ }^{[3]}$.

\section{Public-private cooperation for problem solution}

Currently, mercury-in-glass and clinical electronic thermometers are designated "specified measuring instruments" under the Measurement Law administered by the Ministry of Economy, Trade and Industry. Instrumental error test (verification test) is conducted for all thermometers along with pattern approval test under government control. In contrast, testing and inspection according to technical guidelines of respective companies are conducted under responsibility of the thermometer manufacturers, for maintaining the reliability of temperature readings for newly developed and marketed ear thermometers.

Ear thermometer attracted attention for its usability, and its use spread immediately after it became commercially available. On the other hand, complaints from users increased concerning its performance and reliability including differences in measurement principle and usage compared to conventional clinical thermometers. In 1998, the National Consumer Affairs Center of Japan, a public institution that conducts surveillance study from standpoint of consumer protection, reported "Attention! Ear Thermometers Tend to Measure High", ${ }^{[4]}$ and subsequently newspapers and magazines wrote that "the temperature readings show large variation" or "measurement is higher than actual temperature". ${ }^{[5]}$

In such a situation, "Survey and Research Committee for New Clinical Thermometer" was established in 1998 with members from the government (former Ministry of International Trade and Industry; former Ministry of Health and Welfare), medical professions, consumer organizations, clinical thermometer manufacturers, AIST (formerly National Research Laboratory of Metrology, Agency of Industrial Science and Technology). The committee conducted questionnaire survey to manufacturers and retailers of clinical thermometers, medical institutions, and consumers, to study the situation in Japan. According to this survey, while manufacture and sales of ear thermometer reached nearly 1 million per year, it became clear that neither medical specialists nor consumers had sufficient understanding of performance, measurement principle, usage, or reliability of ear thermometers ${ }^{[2]}$.

With cooperation of the committee members, AIST checked the temperature readings of ear thermometers in the market using an existing simple blackbody furnace, and found that some types of thermometers presented bias and large variations of $0.5^{\circ} \mathrm{C}$ or more in temperature readings.

Through this survey, two points were highlighted as technical issues arising from rapid market expansion of new ear thermometers:

(1) Provision of sufficient information to users about measurement principle and proper usage

(2) Establishment of technical standard, and traceability system for calibration and conformity assessment of ear thermometer

Of the above two points, (1) was an issue mainly for manufacturers, retailers, and industry, while immediate effort by the government centering on AIST was strongly in demand for (2).

The committee also conducted survey for the status of standardization,measurement standard and traceability ${ }^{\text {Term }}$ ${ }^{4}$ for ear thermometers. Work on ear thermometer was done primarily in the United States, which was first in the world to market the product, and Germany, where it diffused widely after acquiring the technology from the U.S. In 
the United States, industrial standard was established by American Society for Testing and Materials (ASTM) ${ }^{[6]}$, while national measurement standard system was slow to develop. In Europe, the preparation was in progress for the European Norm $(\mathrm{EN})^{\text {Term } 5}$ lead by Germany that was center for major manufacturers ${ }^{[7]}$ of ear thermometers. In Germany, Physikalisch-Technische Bundesanstalt (PTB), a national metrology institute, actively engaged in the technical development of measurement standards for ear thermometers.

\section{Setting research goals and scenario for achievement}

As issues to be tackled by AIST described in the previous section, establishment of traceability system for ear thermometers to satisfy user demand was set as a goal, and it was also desirable to utilize the results of technical development obtained in the process of achieving the research goal for performance assessment of ear thermometers. Specifically, $0.2{ }^{\circ} \mathrm{C}(95 \%$ confidence level) was set as the technical goal for uncertainty of temperature readings (measurement result) in ear thermometers at a commercial level. Since the maximum permissible error for measurement methods for mercury-in-glass and clinical electrical thermometers was set at $0.1{ }^{\circ} \mathrm{C}$, some users requested $0.1{ }^{\circ} \mathrm{C}$ uncertainty for ear thermometer. However, estimating the currently existing technical level of ear thermometers on market, and considering the fact that $0.2{ }^{\circ} \mathrm{C}$ uncertainty was employed in drafts of the industrial standards ${ }^{[6,7]}$ in the US and Germany, the Survey and Research Committee for New Clinical Thermometer concluded that the goal for Japan should be $0.2{ }^{\circ} \mathrm{C}^{[2]}$.

To achieve this goal, AIST considered the necessary essential technology and scenario as shown in Figure 2. Research goals of AIST were set as follows: (1) development of performance test technology for conformity assessment ${ }^{\text {Term } 6}$, (2) development of calibration technology for radiance temperature scale of ear thermometers for accuracy management, and (3) establishment of national primary standard for radiance temperature scale that serves as basis of traceability system. To meet these goals, AIST started "the development of high-precision blackbody radiator (standard blackbody furnace)" as a common and key technology. In the development of blackbody radiator (BBR), elemental technologies were designated as follows: (A) technology for precise thermostatic fluid bath needed to realize stable and uniform temperature field, (B) technology for quantitative assessment of radiant property of blackbody cavity, and (C) design and manufacture technology to realize optimal blackbody cavity for calibration of ear thermometers.

\section{Adoption of measurement management system for Japan}

Figure 3 shows how temperature scale of ear thermometers leads to the national primary standard via chain of calibrations (the traceability system) from the viewpoint of technical practicability. There were major technologies to be developed in each phase of traceability, and an issue was to whom the responsibility for the technical work at each phase would be assigned in the social system of metrological management. For calibration and assessment of ear thermometer, which is based on the principle of infrared radiation thermometry, BBR emitting ideal blackbody radiation traceable to correct temperature scale was necessary. The main component of BBR is a reference thermometer that provides standard temperature scale and a blackbody cavity that is a source of thermal radiation and a thermostatic fluid bath. Therefore, we considered what should be the most appropriate system under which each component could be managed to control the quality of the BBR.

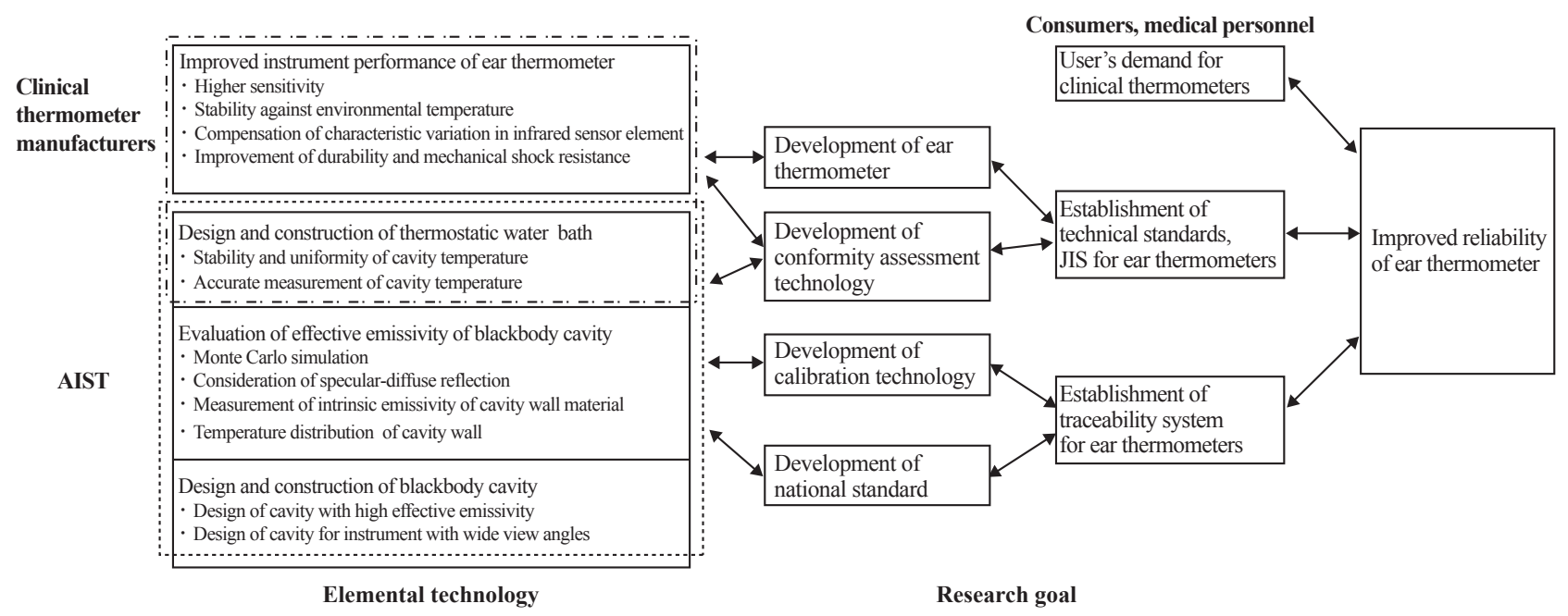

Fig. 2 Research goal and scenario for improving reliability of ear thermometers 
In general, ear thermometer manufactured by companies is calibrated against the working standard BBR available in the company. The working standard BBR is also calibrated against the higher level standard BBR, and this standard $\mathrm{BBR}$ is traceable to national standard for temperature with high-precision contact thermometer. Practical BBR for body temperature range requires a reference thermometer to accurately determine the temperature of blackbody cavity, and a high-precision platinum resistance thermometer (PRT) can be used. For this PRT sensor, calibration system (traceability system) based on the national standard in AIST had already been established and is actually in operation. Using this traceability system, it is possible to calibrate the temperature scale at sufficiently small uncertainty level of less than $0.01{ }^{\circ} \mathrm{C}$ for the reference thermometer.

If the goal value of uncertainty of clinical thermometer reading is $0.2{ }^{\circ} \mathrm{C}$, the uncertainty of working standard BBR, which will be used for calibration and assessment for clinical thermometer of a manufacturer should be approximately $1 / 3$ or less (in this case $0.07{ }^{\circ} \mathrm{C}$ or less) of uncertainty for the clinical thermometer. Moreover, the higher-level standard BBR must have even smaller uncertainty (for example, $0.04{ }^{\circ} \mathrm{C}$ or less) than the working standard BBR of the manufacturer.

Next, various technical works are necessary at each phase of a chain of calibration as shown in Figure 3, and a choice must be made on which sector will be responsible for these works. As shown in the lower part of Figure 3, after careful comparative review of the three potential measurement management systems, the system that matched the current technological and social situations was selected.
(1) Testing and inspection are done based on verification scheme of the Measurement Law (measurement management system in which verification of all products is conducted under government authority through law enforcement).

(2) Traceability of manufacturers' working standard BBR to national standard for temperature (high-precision standard PRT sensor at AIST) is required by industrial standard, and specification of working standard BBR is also established by industrial standard (measurement management system totally dependent on autonomous activity of manufacturers in industry).

(3) The government (AIST) provides new calibration service for radiance temperature scale ${ }^{\text {Term } 7}$, while traceability to AIST radiance temperature standard is established by industrial standard for manufacturer's working standard BBR (measurement management system in which responsibility is shared by government and manufacturers in industry).

The following discussion and selection were done for each of the above measurement management systems.

\subsection{Measurement management system based on verification of Measurement Law}

The Measurement Law in Japan designates measuring instruments that are particularly important to economic activities and services as "specified measuring instrument", and also requires a pattern approval test for structure and specification of the measuring instrument as well as a test of instrumental error for each measuring instrument. In case of clinical thermometer, conventional mercury-in-glass and electronic thermometers have been manufactured and sold after being designated as specified measuring instruments by the Measurement Law. Under the measurement

Level of uncertainty
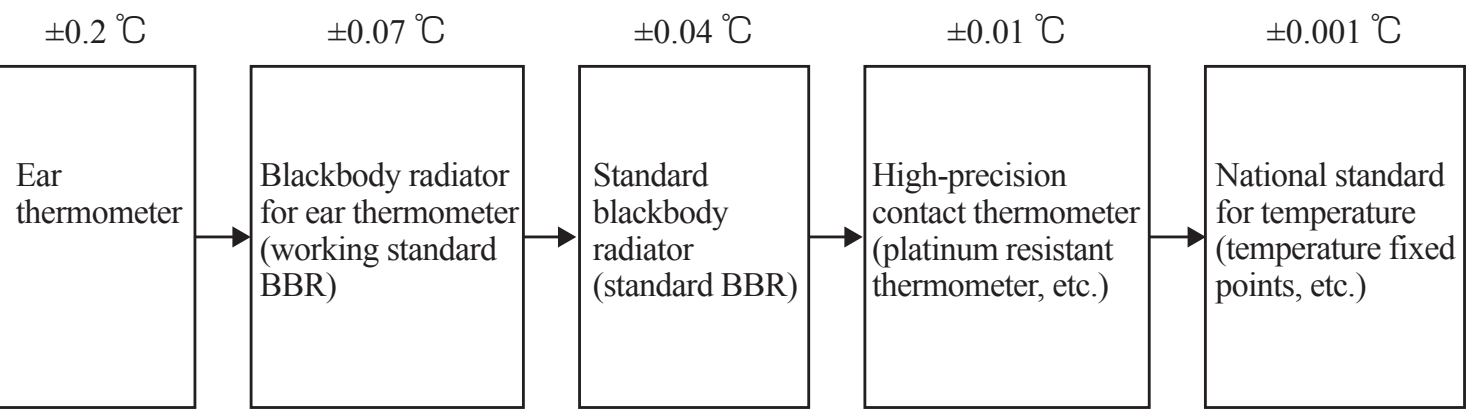

Choice(1)

Verification by Government

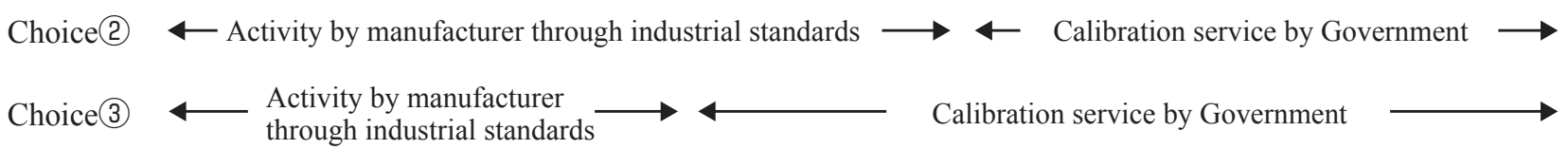

Fig. 3 Traceability and measurement management system of ear thermometer 
management system of the Measurement Law, the main body of management is the government (Ministry of Economy, Trade and Industry) that administers the law, and testing and inspection are also conducted according to rules set by the Measurement Law. All clinical thermometers sold on the market are guaranteed by the government to have certain level of specification and accuracy designated by law, and consumers and users, who may not have technical knowledge of the product, can expect to be able to purchase and use a measuring instrument of a certain quality.

In case measurement management is based on Measurement Law, detailed rules and standard must be determined for procedures and equipments for calibration and assessment. Although this scheme is extremely important from the point of fairness and openness of calibration and assessment, the manufacturer's technological autonomy for measurement management may decrease accordingly. This may become an obstruction for improving production performance (accuracy) through new technical development as well as new product development. In other words, management by Measurement Law is effective in case the methods and equipment for calibration and assessment are commonly available and the product technology has already matured.

Reviews were done by the Survey and Research Committee for New Clinical Thermometer and subsequent JIS Committee established in 2000 for the adequacy of designating ear thermometers as specified measuring instruments. As a result, for ear thermometers, it was concluded that designation of a specified measuring instrument by Measurement Law should be left as a matter of future deliberation for the following reasons: (1) a common method and equipment of calibration and assessment are not widely available among manufacturers, and (2) the product technology is still in a development phase, so early regulation by law has the risk of withholding the advancement of product technology in future.

\subsection{Measurement management system by industrial standardization}

Measurement management system based on autonomous activity of manufacturers by technical standardization (industrial standard document) is thought to be on the opposite end of enforced measurement management by Measurement Law. High quality working standard BBR can be realized by manufacturers by establishing composition and specification of the practical BBR accordong to an industrial standard document such as JIS, and by sharing essential technology including quality management process. In addition, by using contact thermometer, for which traceability to the national standard is established, as reference thermometer, it can be expected that the manufacturers will conduct calibration and assessment at a certain target level or higher.
If such measurement management system can be introduced, merits include: (1) the government sector will not have to take on more work because it will not be required to launch a new calibration service, and (2) compared to regulation by the Measurement Law (Section 4.1), improvement of calibration and assessment technology and standard equipment can be done flexibly and easily to accommodate future product developments, and therefore will not obstruct development and efficiency of the manufacturers' own calibration and assessment technology. On the other hand, total dependence on autonomous system of industrial standard does not have the force of regulation by law, so introduction and operation of technology and equipment will be done under the responsibility of the manufacturers of clinical thermometers. Moreover, since sharing of technological information including standard facility will be limited almost entirely to documented information, there is a risk that it may not be applied appropriately in practice.

To review the adequacy of the above measurement management system from a technical aspect, AIST conducted questionnaire survey on standard facility and measurement management approach used by manufacturers and retailers of ear thermometers in Japan through opportunities such as Standardization Committee, and also actually visited the manufacturers. According to the result, many manufacturers already had large-scale manufacturing and inspection processes for ear thermometers, and many had already introduced and were operating standard equipment (working standard BBR) created on their own. Therefore, in the situation at the time, even if AIST led the standardization of working standard BBR, it was determined that, for economic reasons, it would be difficult for the companies to replace the standard equipment immediately. Moreover, manufacturers of ear thermometers overall had high technology for mercury-in-glass thermometers and thermistor electronic thermometers, but did not have advanced technological experience in managing BBR for ear thermometers, which differed greatly in principle. Therefore, it was concluded that maintaining long-term reliability of ear thermometer in the Japanese market under measurement management system by industrial standardization only was not practical at the time.

\subsection{Measurement management system based on calibration service for radiance temperature scale by the government}

Based on the conclusions of Sections 4.1 and 4.2, a measurement management system in which AIST provides new calibration service for radiance temperature scale of working standard BBR in industry was considered. This system is positioned between the aforementioned two cases.

In case of Section 4.2, traceability to national standard is maintained for the reference contact thermometer of working standard BBR, while the blackbody cavity is regulated by 
industrial standard (technical standard document). Here, direct traceability to national standard is maintained for radiance temperature measured by the ear thermometer. For radiance temperature scale, existing calibration service is not yet organized as in contact thermometers, so the government must take some responsibility for design and establishment of new traceability system for ear thermometers. However, if it becomes possible for AIST to provide direct calibration service of radiance temperature scale of working standard BBR, the clinical thermometer manufactures will be able to make the uncertainty estimation of the calibration and conformity assessment, without necessarily having advanced technology or knowledge for the structure or operation of working standard BBR, because they can rely on the high technological expertise of AIST (the government). Also, the manufacturers may develop their own technology including working standard BBR, and may be able to utilize the equipment they currently own. Therefore, this method was adopted because in current technological and social situation, the cost to the government can be minimized while maintaining high reliability of ear thermometers .

\section{Development of national standard for radiance temperature}

Urgent issue for AIST after choosing the measurement management system described in Section 4.3, was the development of national standard for radiance temperature, which will serve as the primary standard for the ear thermometer. It would be preferable if results of these technical developments can also be used widely in performance tests including calibration of ear thermometers in industry.

Conventional mercury-in-glass and electronic clinical thermometers are contact thermometers in which the sensor probe is placed in contact with the human body site to reach thermal equilibrium state to measure the temperature.

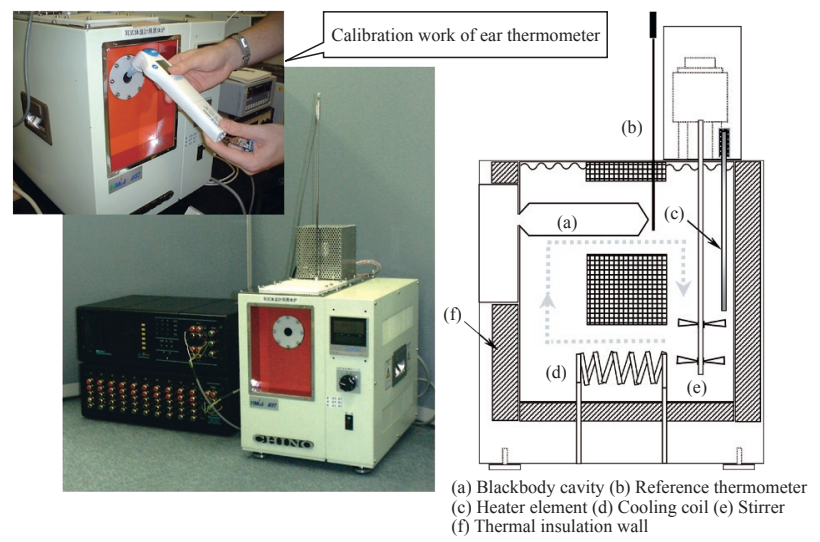

Fig. 4 Standard BBR for calibration of ear thermometer developed by AIST (external appearance and cross sectional view)
Therefore, they can be calibrated or assessed by placing them in a thermostatic fluid bath using high-precision liquid-inglass thermometer or platinum resistant thermometer as a reference thermometer. Also, evaluation of uncertainty of calibration and management of calibration equipment can be done relatively easily.

In contrast, ear thermometer is an infrared radiation thermometer that measures radiance in the infrared wavelength region and estimates body temperature based on Planck's law. Therefore, it is technically impossible to directly compare temperature scale against contact thermometer such as liquid-in-glass or platinum resistant thermometers. Calibration and assessment of infrared radiation thermometer must be conducted against standard "blackbody radiator (blackbody furnace)" that generates ideal thermal radiation (blackbody radiation) according to Planck's law. The authors have been working on the development and characterization for high-precision blackbody radiation source in room temperature region, and they started the development of standard BBR especially for calibration of ear thermometer based on these elemental technologies.

Figure 4 shows the standard BBR for calibration of ear thermometer developed by AIST. High-precision platinum resistant thermometer was employed as standard of temperature scale (reference thermometer), and metal blackbody cavity was installed horizontally in precise thermostatic water bath. When standard BBR realized the radiance temperature scale, the components of uncertainty could be classified as follows:

(1) Uncertainly of calibration of reference thermometer

(2) Uncertainty of cavity temperature measurement by reference thermometer

(2)-1 Uncertainty of temperature measurement of water in bath

(2)-2 Difference between measured temperature of water and temperature of inner surface of cavity (heat loss effect of cavity)

(3) Effective emissivity of blackbody cavity (difference from ideal value 1)

(3)-1 Cavity emissivity of blackbody cavity under isothermal condition

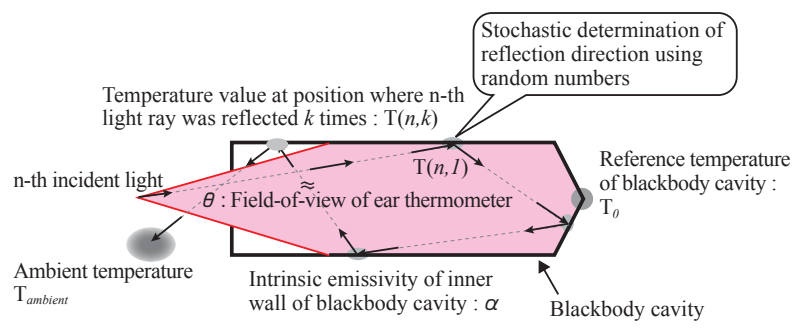

Fig. 5 Evaluation of effective emissivity of blackbody cavity by Monte Carlo simulation 
(3)-2 Effect of temperature distribution of cavity wall (4) Stability and reproducibility of the system

Compared to conventional type of industrial infrared radiation thermometer, ear thermometer has much wider field-of-view, so accurate calibration cannot be achieved when ordinary shape of cavity designed for thermometers with small view angle is used. Therefore, a shape of cavity with sufficiently high effective emissivity for ear thermometer having wide-angle was newly designed based on the Monte Carlo simulation ${ }^{[8]}$. Figure 5 is a schematic diagram of the Monte Carlo simulation. Light ray bundles were entered into the cavity from outside of aperture opening, the reflection on wall surface was simulated using random numbers, and the effective absorptivity (corresponds to effective emissivity) of the cavity was calculated from the probability of absorption of the incident ray bundle in the cavity. The reflective property of the cavity wall was expressed by a model composed from perfect diffuse reflection and specular reflection. Then, the effect of temperature distribution on the cavity wall was also quantitatively evaluated by inputting measured temperature distribution in the water bath to the simulation.

Intrinsic emissivity of coating material of cavity wall was measured with a Fourier transform infrared spectrometer system developed at AIST. The measurement uncertainty of spectral emissivity data was evaluated at $1 \%$ or less $^{[9]}$, and was taken into account as an uncertainty of intrinsic emissivity of cavity wall. Measured emissivity data was used as parameter for Monte Carlo simulation to evaluate the effective emissivity of cavity. Also, effect of heat loss by convection of air and thermal radiation in the blackbody cavity was evaluated using high-resolution infrared radiation thermometer ${ }^{[10]}$.

Table 1 shows the performance (uncertainty budget) of standard BBR, which is the national standard for radiance temperature scale developed by AIST. For reference

Table 1. Uncertainty budget of standard BBR developed by AIST

\begin{tabular}{|l|c|c|c|c|}
\hline & \multicolumn{3}{|c|}{ Temperature of blackbody cavity } & Unit \\
\hline & $32^{\circ} \mathrm{C}$ & $37{ }^{\circ} \mathrm{C}$ & $42^{\circ} \mathrm{C}$ & \\
\hline Component of uncertainty & \multicolumn{3}{|c|}{ Uncertainty } & $\mathrm{mK}$ \\
\hline Calibration of reference thermometer & \multicolumn{2}{|c|}{5} & $\mathrm{mK}$ \\
\hline $\begin{array}{l}\text { Temperature measurement by } \\
\text { reference thermometer } \\
\text { (including stability of water bath) }\end{array}$ & \multicolumn{2}{|c|}{5} & $\mathrm{mK}$ \\
\hline Heat loss inside cavity & \multicolumn{3}{|c|}{$<1$} & $\mathrm{mK}$ \\
\hline Effective emissivity of isothermal cavity & 8 & 12 & 16 & $\mathrm{mK}$ \\
\hline $\begin{array}{l}\text { Effect of temperature distribution } \\
\text { of cavity wall }\end{array}$ & \multicolumn{2}{|c|}{2} & $\mathrm{mK}$ \\
\hline $\begin{array}{l}\text { Effect of change in ambient temperature } \\
\left(\mathrm{T}_{\text {ambien }}=23 \pm 2 \text { C }\right)\end{array}$ & \multicolumn{2}{|c|}{2} & $\mathrm{mK}$ \\
\hline Combined standard uncertainty & 11 & 14 & 18 & $\mathrm{mK}$ \\
\hline $\begin{array}{l}\text { Extended uncertainty } \\
(95 \% \text { confidencelevel })\end{array}$ & 22 & 28 & 36 & \\
\hline
\end{tabular}

thermometer, about $5 \mathrm{mK}$ uncertainty was maintained by calibrating the standard platinum resistant thermometer against AIST's national standard temperature fixed point cells, and the reference temperature of the cavity was measured at around $5 \mathrm{mK}$ level of uncertainty in the thermostatic water bath shown in Figure 4. Effective emissivity of 0.9995 or more was achieved for the cavity by designing the cavity shape suitable for ear thermometers with wide view angles, and uncertainty of radiance temperature resulting from this was set at $20 \mathrm{mK}$ or less. With these technological developments, radiance temperature scale traceable to the international temperature scale (SI unit for temperature) at approximately $0.03{ }^{\circ} \mathrm{C}$ uncertainty $(95 \%$ confidence level ) was achieved for human body temperature range $\left(32{ }^{\circ} \mathrm{C} \sim 42{ }^{\circ} \mathrm{C}\right)^{[11]}$.

The technology developed for standard BBR was also employed as recommended specification for standard equipment in JIS for ear thermometers which will be explained later ${ }^{[12]}$. Currently, it is commercially available as working standard BBR for body temperature range from a manufacturer which participated in this joint development ${ }^{[13]}$.

\section{Calibration of working standard BBR in industry against the national standard BBR at AIST}

Next, specific calibration method was reviewed to link between the national standard BBR of AIST and working standard BBR of manufacturers in this new measurement management system. As mentioned in Section 5, AIST developed the standard BBR as national primary standard for radiance temperature scale, and the actual calibration will be done by either transporting the manufacturer's working standard BBR to AIST to be calibrated against national standard BBR or by transporting standard BBR of AIST to the manufacturer to calibrate the working standard BBR.

\subsection{Uncertainty estimation for calibration of working standard BBR}

Simulated calibration experiment was conducted to estimate the uncertainty when working standard BBR was calibrated against national standard BBR at AIST. Aside from standard $\mathrm{BBR}$, which is the national standard, a BBR for simulated calibration was prepared at AIST. Four types (about three thermometers for each type) of high-resolution (displayed temperature resolution $0.01{ }^{\circ} \mathrm{C}$ ) ear thermometers provided by several manufacturers were used for direct comparison measurement of radiance temperature of the blackbody cavities. In the experiment, AIST standard blackbody furnace and BBR for simulated calibration were run simultaneously, and were stabilized at nearly identical temperature for calibrating the radiance temperature (e.g. $37.0^{\circ} \mathrm{C}$ ). While monitoring the temperature of each reference thermometer, the difference in radiance temperature between the two BBRs 
was measured using the high-resolution ear thermometers. Comparison measurements were repeated about 10 times for each thermometer, and after evaluating the average and variance of radiance temperatures, the temperature difference of reference thermometer was corrected and the calibration results were calculated.

Figure 6 shows the results of the verification experiment. The average values of radiance temperature differences of the two BBRs were almost zero within the range of variation. However, the variation (standard variation) of calibration values was around $0.03{ }^{\circ} \mathrm{C}$. This variation in value was uncertainty factor that occurred additionally in radiance comparison calibration of the BBR. From these results, approximately $0.06{ }^{\circ} \mathrm{C}(95 \%$ confidence level) was obtained as uncertainty of calibration result in combination of uncertainty of radiance temperature scale of the national standard BBR with the uncertainty of direct comparison measurement with ear thermometers with high resolution mode ${ }^{[1]]}$. This uncertainty satisfied the uncertainty level required for calibration of working standard BBR (approximately $0.07^{\circ} \mathrm{C}$ or less).

\subsection{Verification of uncertainty of calibration by collaborative experiment with manufacturers}

Joint measurement experiment was conducted by AIST and ear thermometer manufacturers to verify the uncertainty level estimated in the simulated calibration experiment as mentioned in the previous section, as well as the validity of the proposed calibration scheme. For this round robin experiment, AIST constructed a standard BBR that could be transported. The standard BBR was transported to seven Japanese thermometer manufacturers by commercial carrier, and the differences with radiance temperatures of working standard BBRs of the companies were measured by the direct comparison method mentioned above.

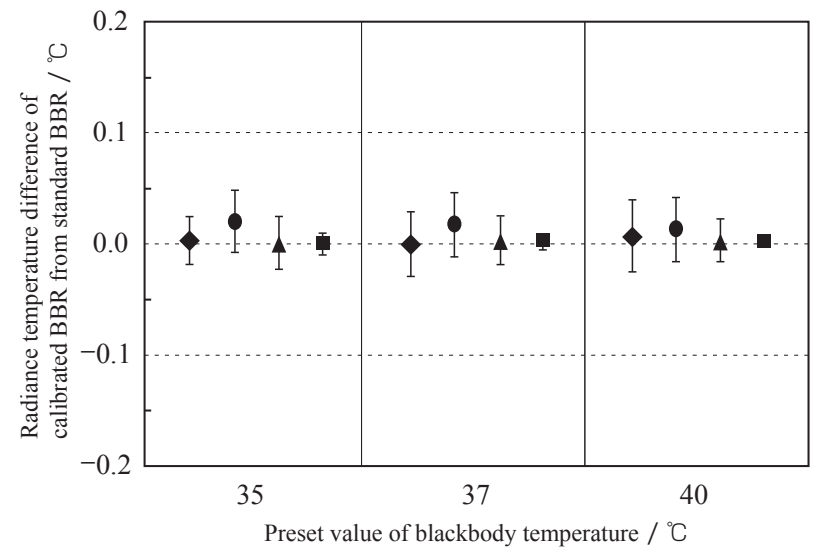

Fig. 6 Verification result for calibration of radiance temperature scale of working standard blackbody furnace against national standard BBR at AIST
Figure 7 shows the measurement results ${ }^{[14]}$. The results of the comparison measurements at each manufacturer have variations of about $0.03{ }^{\circ} \mathrm{C}$, but it was confirmed that the radiance temperature scale of working standard BBR of each company agreed with the standard BBR of AIST within $0.05{ }^{\circ} \mathrm{C}$. This showed that the radiance temperature of working standard BBR of thermometer manufacturers could be calibrated at uncertainly of $0.07{ }^{\circ} \mathrm{C}$ or less, and it was concluded that requirement for calibration and assessment of ear thermometer was met sufficiently based on this traceability scheme.

\section{Calibration service system and verification of international equality}

In establishing traceability system, along with development of national standard and dissemination of temperature scale, an important issue is to verify whether the Japanese national standard and calibration service are equivalent to those of other countries. For this purpose, quality management system in accordance with ISO/IEC 17025 standard ${ }^{\text {Term } 8}$ was organized for calibration service of working standard BBR for ear thermometer conducted at AIST. By operating the quality management system, the quality of calibration service can be maintained based on third party approval.

To verify equivalence of the national standard developed by AIST to the national standards of other countries, international comparison measurements were conducted among national metrology institutes of Germany and United Kingdom. Figure 8 shows the result of the international comparison measurement conducted between PhysikalischTechnische Bundesanstalt (PTB) of Germany and National Physical Laboratory (NPL) of the UK ${ }^{[15]}$. The standard BBRs of AIST and PTB were transported to NPL, and radiance temperature scales were compared directly. From the

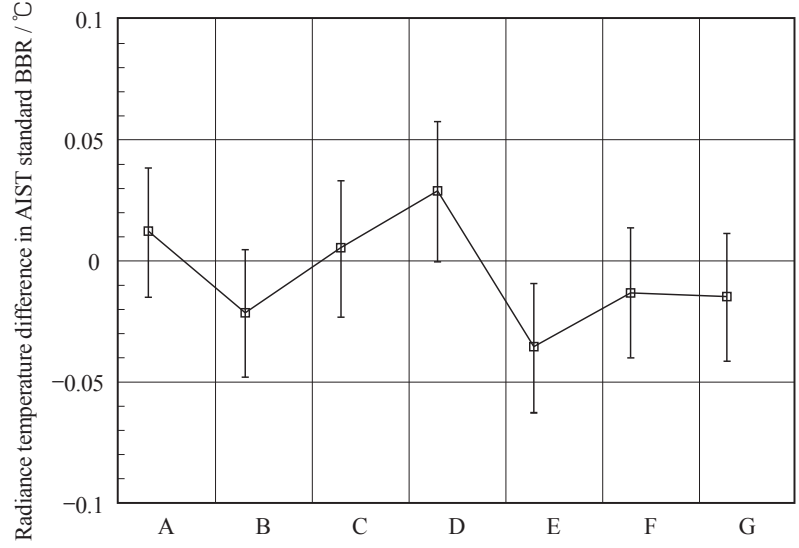

Fig. 7 Result of round-robin comparison measurement of working standard BBRs of clinical thermometer manufacturers with AIST standard BBR

Blackbody temperature is $37^{\circ} \mathrm{C}$. $\mathrm{A} \sim \mathrm{G}$ are participating manufacturers. 
results of this international comparison, it was confirmed that radiance temperature scale realized on each national standard agreed well within the uncertainty level claimed by each institute (about $0.03{ }^{\circ} \mathrm{C}$ ). The result of this international comparison measurement was the first report in the world for standard BBRs for clinical infrared thermometers, and it became a model case for later international comparative measurements in this field. In the Asia-Pacific region, AIST has also conducted a similar international comparison with National Measurement Institute of Australia (NMIA) and obtained satisfactory measurement results.

\section{Summary of research results}

AIST conducted research and development as the national metrology institute to improve reliability of new clinical infrared ear thermometers, and obtained the following results.

- Developed national standard (standard blackbody radiator) with high precision (uncertainty level $0.03{ }^{\circ} \mathrm{C}$ )

- Designed and established new traceability system for radiance temperature for calibration of working standard BBR. Uncertainty level of calibration achieved was $0.06{ }^{\circ} \mathrm{C}$.

- Verified international equivalence of national standard by conducting international comparison measurement with foreign national metrology institutions. Also, maintained quality of calibration service in accordance with ISO 17025 with third party approval.

These results are applied to measurement management system for maintaining reliability (uncertainty level 0.07 ${ }^{\circ} \mathrm{C}$ ) required for calibration and assessment of clinical thermometers, while providing autonomous activity for standard equipment of thermometer manufacturers. They contribute to the improvement of reliability of temperature readings of ear thermometers in the Japanese market.

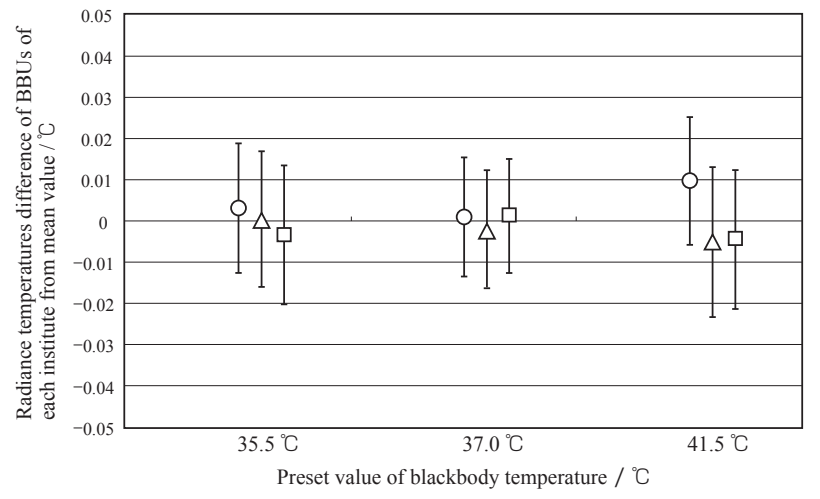

Fig. 8 Result of international comparison of national standard BBR in body temperature region $\bigcirc$ AIST $\triangle$ NPL UK $\square$ PTB Germany

\section{International cooperation to prevent spread of SARS}

Development of standard blackbody radiator and calibration technologies for ear thermometer by AIST was an advanced effort in the world at the time, and the technical achievements are highly evaluated by other countries. In 2003, severe acute respiratory syndrome (SARS) occurred in the Asian region and developed into international crisis. With request of national metrology institutions of Singapore and Taiwan, the standard BBRs and technology developed by AIST were provided swiftly, and enabled to make calibration and assessment of clinical infrared thermometers for screening patients with fevers at airports and seaports. This activity was highly appreciated as a case of international research cooperation of measurement standard technology in a serious social problem that threatens safety and security ${ }^{[3]}$.

\section{Conclusion}

The efforts to establish traceability system and to disseminate standard technology for new ear thermometer were described. Development and dissemination of new measurement standard were conducted to make ear thermometers more reliable to users and to strengthen international competitiveness of Japanese manufacturers for clinical infrared thermometer that has totally a different measurement principle from conventional types of clinical thermometers. AIST developed the world's highest quality national standard based on advanced radiation temperature standard and infrared measurement technology that had been accumulated at AIST, and built new traceability system so high quality standard can be used widely by industry and users. These results contributed greatly to improve reliability of body temperature measurement through calibration service and standardization process by AIST.

\section{Acknowledgement}

The author is grateful for the support and cooperation by Tomoko Fukuzaki of National Institute of Metrology, as well as many persons in and outside AIST concerned in developing this research.

\section{Terminology}

Term 1. Radiance : Amount of radiation energy emitted in specific direction by unit time, unit area, and unit solid angle, for light emission from light source. Unit $\left[\mathrm{W} \cdot \mathrm{sr}^{-1} \cdot \mathrm{m}^{-2}\right]$.

Term 2. Planck's law of radiation: Law of physics governing the thermal radiation characteristic (relationship of temperature and spectral radiation energy distribution) of ideal blackbody. It was formulated by Planck in 1900 . Since emissivity is less than 1 in 
actual body, radiation characteristic is Planck's law multiplied by emissivity value of the surface.

Term 3. Emissivity: Index expressing an optical property of thermal radiation source. Emissivity is 1 in case of ideal blackbody, and actual thermal radiation source have emissivity value between 0 and 1 . In general, emissivity of material surface changes depending on material as well as wavelength, angle, and surface roughness.

Term 4. Traceability: General term for measurement management system from national standard or international standards to user-level measuring instrument through chain of comparisons (calibrations).

Term 5. European Norm (EN): Also known as European Standard. Uniform standard for Europe issued by the Comité Européen de Normalisation (CEN) and Comité Européen de Normalisation Electrotechnique (CENELEC), which are expert committees of the European Union.

Term 6. Conformity Assessment: Act of checking whether product, service, or process satisfy the required standards or directives. It may also be used as general term for testing, inspection, and assessment procedures.

Term 7. Radiance Temperature: Temperature calculated from radiance value at any wavelength region when light source is assumed to be ideal blackbody in accordance with Planck's law. In case light source is ideal blackbody, it corresponds to the thermodynamic temperature, but if it is not blackbody (emissivity is less than 1), the radiance temperature will be different from the thermodynamic temperature of light source.

Term 8. ISO/IEC 17025 Standard: International standard document for quality control of services conducted by testing and calibration institutions. Quality control based on this standard and third party approval are conducted for calibration services of physical standards provided by National Metrology Institute of Japan, AIST.

\section{References}

[1] J.Fraden: Medical infrared thermometry (review of modern techniques), Ed. by J.F. Schooley, Temperature Its Measurement and Control in Science and Industry, vol.6, part II, 825-830, Amer. Institute of Physics, New York(1992).

[2] Shingata taionkei no kaihatsu doko ha'aku to gijutsu kijun tekigo ni kakawaru chosa kenkyu hokokusho (Survey and research report on the development trend and technological standard conformity of new clinical thermometer), Japan Measuring Instruments Federation, (1999) (in Japanese).

[3] Taionkei koseiki SARS de kinkyu taiyo (Emergency offer of calibration equipment of clinical thermometers for SARS), Nikkei Business Daily (May 27, 2003) (in Japanese).
[4] Chui! Takameni deru keiko niaru mimi shiki taionkei (Warning! Ear thermometers that tend to measure high), Claim Processing Test, No.5, National Consumer Affairs Center (1998) (in Japanese).

[5] Mainichi Shimbun (April 22, 1998), Asahi Shimbun (April 27, 1998), Kyoto Shimbun (May 1, 1998), Sankei Shimbun (May 7, 1998), Yomiuri Shimbun (May 7, 1998), and other newspapers (in Japanese).

[6] ASTM designation E-1965-98, ASTM committee, E20.20 (1998).

[7] prEN 12470-5, CEN TC205 (2000).

[8] J. Ishii, T. Fukuzaki, T. Fujihara, and A. Ono: Koshiya sekigai hosha ondokei koseiyo kokutai kudo no hosha tokusei hyoka (Evaluation of radiative property of the blackbody cavity for calibration of infrared radiation thermometers having wide view angles), Transactions of the Society of Instrument and Control Engineers, 37, 468-470 (2001)(in Japanese).

[9] J.Ishii and A.Ono: Uncertainty estimation for emissivity measurements near room temperature with a Fourier transform spectrometer, Meas. Sci. Technol., 12, 21032122(2001).

[10] J.Ishii and A.Ono: Low-temperature infrared radiation thermometry at NMIJ, Temperature: Its measurement and control in science and industry, vol.7, ed. D. C.Ripple, AIP, 657-662(2003).

[11] J.Ishii, T.Fukuzaki, T.Kojima and A.Ono: Calibration of infrared ear thermometers, Proc.TEMPMEKO 2001, $607-$ 612(2002).

[12] Japanese Industrial Standard: JIS T-4207:2005 Mimiyo sekigaisen shiki taionkei (Infrared Ear Thermometer), Japanese Standard Association (2005)(in Japanese).

[13] Chino Corporation Product Catalog (in Japanese)

[14] Mimishiki taionkei hyojun kyokyu kenkyukai hokoku (Report of research group for ear thermometer calibration service), Japan Measuring Instruments Federation (2002)(in Japanese).

[15] J.Ishii, T.Fukuzaki, H.C.McEvoy, R.Simpson, G.Machin, J.Hartmann, B.Gutschwager and J.Hollant: A comparison of the blackbody cavities for infrared ear thermometers of NMIJ, NPL, and PTB, Proc. TEMPMEKO 2004, 10931098(2005).

Received original manuscript October 3, 2007 Revisions received November 27, 2007 Accepted November 27, 2007

\section{Author}

\section{Juntaro Ishii}

Completed his Ph.D in physics at Keio University in 1996. Joined National Research Laboratory of Metrology (NRLM), Agency of Industrial Science and Technology in 1996. Senior researcher of (National Institute of Metrology) of AIST in 2001. Head of Radiation Thermometry Section, in 2005. Guest researcher at National Physical Laboratory of UK in 2002. Won APMP Iizuka Prize in 2006. Research fields include temperature standard, radiation thermometry, infrared measurement, and surface temperature measurement. 


\section{Discussion with reviewers}

\section{Future technological issues for improving reliability of ear thermometers \\ Question (Akira Ono)}

The point that excels in this paper is that it describes not only a technological development, but is a comprehensive discussion that also addresses balance of social systems such as verification, calibration service, and industrial standards.

I think the traceability system established according to the result of this study is satisfying to keep the tolerance of ear thermometers to $\pm 0.2{ }^{\circ} \mathrm{C}$. On the other hand, what is the level of long-term stability for the ear thermometers themselves?

Also, which points do you think need $R \& D$ in the future to keep the tolerance to $\pm 0.2{ }^{\circ} \mathrm{C}$ or less for commercial ear thermometers? I would like to hear the author's view on both technological development of thermometer and technological development of measurement standard.

\section{Answer (Juntaro Ishii)}

At AIST, an examination of long-term stability and reproducibility of several commercially available ear thermometers in the market were conducted by periodical calibration over one and a half year against the standard BBR of AIST in 1999. The results showed that most ear thermometers have long-term stability of approximately $0.2{ }^{\circ} \mathrm{C}$ or less, and some ear thermometers go over $0.2{ }^{\circ} \mathrm{C}$ in the period of about half a year, and in some the temperature scale change was as great as $0.4^{\circ} \mathrm{C}$ (Figure a shows the results). It is difficult to specify the cause of the long-term instability, but in general, they are due to the drift of infrared sensor responsivity, the change in characteristic of compensation temperature sensor, and the deterioration of the performance of the optical element.

If the goal is to keep the tolerance of the commercial ear thermometer to $0.2{ }^{\circ} \mathrm{C}$ or less, I think it is necessary to employ design and specification that can maintain the long-term stability of at least one year against the standard BBR, and this should be the technological development goal of the ear thermometer by manufacturers.

Moreover, for the metrological control of clinical thermometers used in medical institutions, regular performance check about once a year may be effective, in addition to the initial assessment when products are shipped out. For such a regular check, it is necessary to develop and spread a practical BBR calibrator that can be used at medical institutions and thermometer dealers.

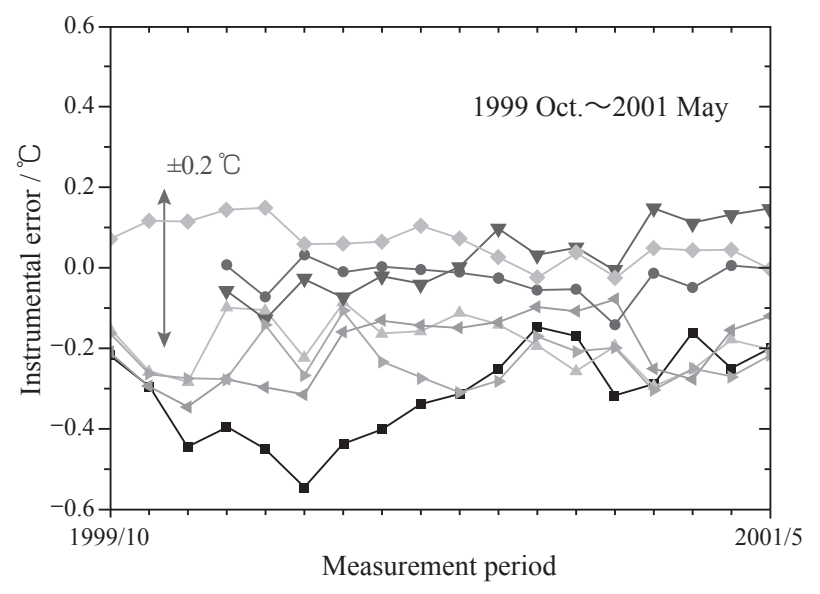

Fig. a Long-term stability of temperature scale of commercial ear thermometers
Also, as discussed in the paper, the uncertainties that occur during the calibration of working standard BBRs by the comparison measurement method are relatively large (variation is about $0.03{ }^{\circ} \mathrm{C}$ by standard deviation) in the present traceability scheme of radiance temperature, and we are aware that it is difficult for the thermometer manufacturer to conduct hierarchical organization of working standard BBRs within the company. For this, I think a technological development is necessary for conducting radiance comparison measurement at smaller uncertainty using standard BBRs. Until now, commercial high-resolution ear thermometers were applied as a radiance comparator, but currently, we are working on the development of high-performance infrared radiation thermometer with small measurement variation compared to the ear thermometers, and are obtaining good experiment results toward realization of the radiance comparison measurement at smaller uncertainly of around $0.01{ }^{\circ} \mathrm{C}$.

\section{Other methods for calibration of working standard BBR} Question (Akira Ono)

I think as a method for calibrating working standard BBRs of thermometer manufacturer against national standard BBR of AIST, there is a method of using infrared ear thermometer itself as the transfer (traveling) standard, other than a direct comparison of blackbody cavities described in the article. Also, I think there is a method of removing the blackbody cavity from the working standard BBR of thermometer manufacturer, transport it to AIST, and calibrate against the standard BBR. How do you evaluate such calibration methods compared to the direct comparison method? Answer (Juntaro Ishii)

Figure $\mathrm{b}$ shows the transfer standards and calibration schemes for realizing traceability of radiance temperature scale.

- On method of using ear thermometer as a transfer standard

Using ear thermometer as a transfer standard will reduce the cost of transportation compared to a larger BBR system, and for manufacturers, there is a merit that it is relatively easy to calibrate several working standard BBRs using the transfer standard thermometer in the house. However, since the standard radiance temperature scale is realized and maintained on ear thermometer which will serve as a transfer standard, superior transport stability and long-term stability shall be required in addition to the basic performance such as the temperature resolution.

AIST conducted experimental verification for calibration scheme of using ear thermometer as a transfer standard along with direct comparison scheme by transporting BBR. According to the experiment, in the scheme using thermometer as transfer standard, the level of variation of calibration increased to 0.05 ${ }^{\circ} \mathrm{C} \sim 0.1{ }^{\circ} \mathrm{C}$. This large variation occurred due to the level of stability and reproducibility of ear thermometer during traveling,

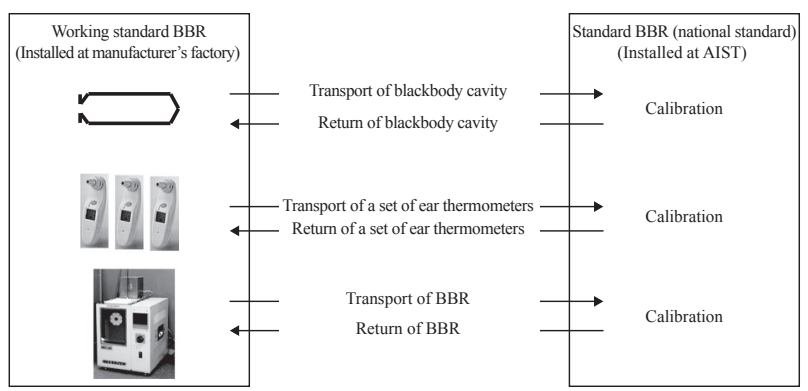

Fig. b Options of transfer standard 
and was directly reflected in the uncertainty of calibration of working standard BBR. Therefore, since the realization of calibration uncertainty of $0.07{ }^{\circ} \mathrm{C}$ or less, which was set as a development goal, became difficult to achieve, we could not employ this calibration scheme. I think this method should be reconsidered if the transfer standard (radiation thermometer) with a higher stability and reproducibility can be successfully developed in the future.

\section{- On method of transporting blackbody cavity}

In case of BBR for ear thermometer a blackbody cavity which is thermal radiation source is placed in a thermostatic water bath with sufficiently good temperature uniformity and stability, and temperature of water near the bottom of the cavity is measured using calibrated a reference thermometer. With the thermostatic fluid bath, it is possible to realize temperature uniformity of about $0.01{ }^{\circ} \mathrm{C}$ using devices available commercially, and for long-term management of performance, parameters such as the temperature distribution in the water bath can be checked regularly using a contact thermometer with high temperature resolution such as the reference thermometer.

On the other hand, effective emissivity of the blackbody cavity is its performance index which is expected to change greatly not only due to the shape and the material of the cavity but also by the optical property and the deterioration of coating of the inner wall of the cavity, and it is not easy for thermometer manufacturers or testing institutions to quantitatively assess on their own. Therefore, it will actually be possible to calibrate the effective emissivity of blackbody cavity, if it is possible to remove the blackbody cavity from the working standard BBR of the manufacturer, transport it to AIST, install it in the thermostatic water bath of AIST in which the performance is examined, and conduct comparison measurements of radiance temperature with the BBR (blackbody cavity) of national standard. In this case, the client of the calibration service will have a merit of not having to transport large thermostatic water bath system, and will be also able to conduct the long-term measurement management within the company through a group management method by using a set of exchangeable (standard) blackbody cavities calibrated against national standard BBR.

If this calibration scheme could be realized, it would have been a unique and practical traceability system in the world for the effective emissivity of blackbody cavity, but survey showed that existing working standard BBR at thermometer manufacturers have wide variation in shape and specification of blackbody cavity, and there were reports of difficulty in removing and transporting the cavity part in manufacturer's BBR, so we could not put it to practice.

\section{Trend of international standard Question (Akira Ono)}

The main subject of this article was measurement standard for ear thermometer, but standardization (document standard) is also an important point. What is the state of international industrial standards for ear thermometers?

\section{Answer (Juntaro Ishii)}

As mentioned in the paper, JIS for Japan, ASTM for the United States, and EN for Europe are established as product standards for ear thermometer.

From 2005, the work for new international standardization is in progress through a joint proposal by ISO and IEC, mainly for clinical electrical (thermistor) and ear thermometers. For this international standardization, the author is participating in an international working group activity as an expert member. The following committee draft document has been issued as of October 2007.

ISO/IEC CD.2 80601-2-56, "Medical Electrical Equipment Part
2-56, Particular requirements for basic safety and essential performance of clinical thermometers for body temperature measurement", ISO/IEC (2007).

\section{Use of ear thermometer for preventing spread of infectious disease Question (Akira Ono)}

We've been warned that if bird influenza virus mutates and spreads around the world, it will be a major threat to humankind. When SARS epidemic occurred in 2003, the experiences of Taiwan, Singapore, China, and Hong Kong where the infection spread within the country may be valuable for Japan in the future. What do you think are the important points for body temperature measurement in public places? Also what role do you think ear thermometers will play?

\section{Answer (Juntaro Ishii)}

Immediately after SARS epidemic became a social problem in the Asian region in 2003, the author actually visited the metrology institutes in Singapore and Taiwan, and gathered information on case studies for screening infected patients with fever at such as international airports. All countries screened patients with fever of $38.0^{\circ} \mathrm{C}$ or higher, and inspection using infrared thermographic instruments and ear thermometers were conducted.

From these case studies and discussion with persons involved, technical issues in public screening were: (1) maintaining stability of thermography and ear thermometer, and (2) efficient and reliable screening using combination of sensing devices. For (1), in contrast to laboratory with stable environmental condition, it was difficult to maintain reliability of the instrument (temperature reading) in places with severe fluctuations in environmental condition such as humidity and temperature. At the airport in Singapore, screening was done by installing improved thermography system in the passageway to the immigration counter, but the temperature reading of the thermograph image was not sufficiently stable. A simple BBR was placed behind the passageway, so people and blackbody cavity could be observed simultaneously by the thermography to make corrections of drifts of temperature scale of thermograph image. For (2), to conduct efficient and highly reliable screening on a large number of people, preliminary screening using thermography system was conducted, and full inspection using ear thermometer was conducted to people suspected of a fever, and this prevented giving stress to the travelers.

These cases and technical issues would be highly suggestive for operating public screening in Japan.

\section{Shape and surface coating of standard blackbody cavity \\ Question (Akira Ono)}

It is written that the blackbody cavity for ear thermometers

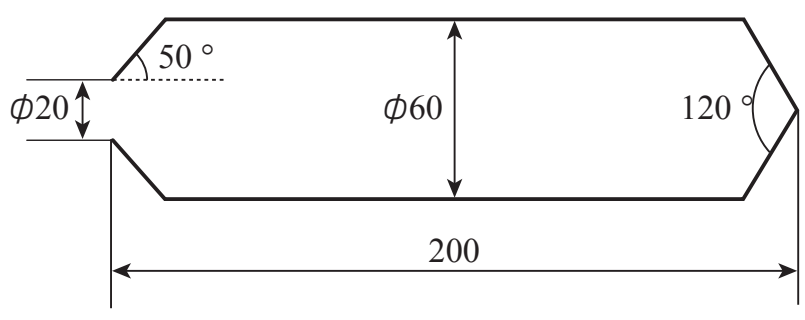

unit:mm

Fig. c Standard blackbody cavity for ear thermometer developed by AIST 
having wide view angles was designed by conducting Monte Carlo simulation, but what is the shape of the cavity recommended by AIST and employed in JIS? Also, what material is used normally to coat the inner wall of cavity of the standard BBR, and what level of intrinsic emissivity does it have in the infrared region?

\section{Answer (Juntaro Ishii)}

Figure $\mathrm{c}$ shows the cross sectional view graph of the standard blackbody cavity for calibration of ear thermometer developed by AIST. The material of the blackbody cavity is oxygen-free copper with high thermal conductivity, and the wall of the cavity is designed so its thickness will be $0.5 \mathrm{~mm}$ or less. The inner wall of the blackbody cavity must be blackened so it will have high emissivity of 0.95 or more in the infrared wavelength region. AIST measured spectral emissivity for black paint and coating material commercially available in Japan and overseas in the infrared region using the FTIR spectrometer system, and we employed black paint (Nextel's velvet coatings) with spectral emissivity of 0.96 or more in the $5 \sim 12 \mu \mathrm{m}$ wavelength region.

\section{History of development of ear thermometer Question (Naoto Kobayashi)}

Ear thermometer is an innovative clinical thermometer that allows non-contact measurement in short time. You mentioned that it was developed by an American company in the 1990s, but was this type of thermometer developed in Japan or other countries at that time? If it was completely original creation by the American company, what is the reason that this company was able to make it while others couldn't? If it was a result of development competition, why couldn't the companies of Japan or other countries win this competition?

Answer (Juntaro Ishii)

The approach of determining temperature of skin and body surface by measuring infrared radiation from human body was a measurement technique available for a long time, and was applied to breast cancer diagnosis using thermography system. Also, measurement of temperature around the tympanic membrane was a subject of "basal body temperature (core temperature)" measurement that was medically important since it was close indication of brain temperature. However, in the conventional method, thin wire temperature sensor such as thermocouple or thermistor was pressed directly against the eardrum, so patients (subjects) felt pain and suffering, and it was a special body temperature measurement method done only by medical specialists.

Although I do not know the details of the history of development of current infrared ear thermometer, I guess that there were two technological points in product realization: development of highly sensitive and low cost infrared sensor, and technology for compensation of effect of temperature change in thermometer caused by the temperature fluctuation in surrounding environment and by contact with human body. I think the basic methodology for infrared ear thermometer was already known, and technical development for product realization was in progress not only in the US but also in Europe and Japan. In the US, stateof-art R\&D were conducted for infrared sensor and precise infrared measurement as core technologies in defense and space fields, and I think the US was able to lead the world in producing highly practical ear thermometer using these advanced infrared technology. Although this is my guess, the clinical thermometer manufacturers of Japan and Europe, which had manufactured mercury-in-glass and thermistor thermometers but did not have technological foundation for infrared measurement, might have been thinking, "Product realization for low-cost highly reliable clinical infrared thermometer that can compete with thermistor thermometers was far ahead in time". Then, an American venture company actually developed competitive ear thermometer, and the product won support from users in the American market. I think, only after that, product development by Japanese and European manufacturers has accelerated.

\section{Performance test of ear thermometer in market Question (Naoto Kobayashi)}

What is the level of reliability of current ear thermometer in market? In the comparison test (for FY2005) of ear thermometer conducted officially by the National Consumer Affairs Center, measurement variation of $0.5-0.7{ }^{\circ} \mathrm{C}$ was reported. Is this result unavoidable in the current situation where there is a maximum $0.4{ }^{\circ} \mathrm{C}$ fluctuation in temperature graduation due to insufficient long-term stability, or is it something that can be improved if the traceability for current ear thermometer gets better? These are points of interest and I would like to hear your thoughts. Answer (Juntaro Ishii)

For user-level reliability of ear thermometer, I am aware of the fact that it does not completely satisfy the users. I think it is necessary to divide the issue into two parts for consideration: (1) performance of thermometer as a physical measurement instrument (thermometer), and (2) performance of thermometer as a medical equipment whose measurement subject is the human body.

For (1), as mentioned in the article, it is possible to verify the reliability of measurement and feed the result back to improve product performance by conducting calibration and conformity assessment using standard BBR traceable to international unit (SI) of temperature for infrared thermometer, and I believe that the result of R\&D by AIST is contributing to the improvement of reliability. Some of the products do still have problems of longterm stability, but I think a more stable thermometer will be developed in the future along with verification and assessment using standard BBR and by maintaining traceability.

In contrast, (2) is a matter of obtaining reliability in measuring the human body, which is "measurement subject with variation", and a different approach is necessary from (1). As mentioned in the article, current ear thermometers have different measurement view angles by types, and the measured site is not necessarily "eardrum" itself, and in many cases it measures "interior of ear canal including eardrum". In general, a nonnegligible temperature difference (temperature distribution) may occur in the eardrum and the surrounding ear canal, and moreover, there may also be differences in emissivity between the eardrum and the skin surface of the surrounding ear canal. Therefore, even if the same subject is repeatedly measured with the ear thermometer, the measured data may show large variation depending on how the ear thermometer probe is inserted into the earhole. Moreover, occurrence of different temperature readings among ear thermometers with different measurement view angles is unavoidable in current circumstances.

These issues cannot be necessarily verified by engineering assessment using "physically correct BBR" as in (1), and it is necessary to increase reliability by conducting the clinical assessment with assumed medical knowledge. From the standpoint of developing the ear thermometer, "thermometer that selectively measures eardrum", and "method of taking repeated number of measurements in one measurement, and then using highest temperature value as measurement result" and "method of displaying values converted to temperature of armpit or oral by processing data based on the characteristics of thermometer and the clinical information rather than using measurement as it" are being researched and developed. On the other hand, in the international standardization (ISO/IEC) which is in progress now, the ear thermometer is categorized as a clinical thermometer 
that requires clinical assessment, clinical assessment with subjects including patients with fever is conducted along with an engineering evaluation by standard BBR, and an assessment of clinical reliability using the statistical method is proposed. In this case, the achievement of uncertainty $0.2^{\circ} \mathrm{C}$ is strictly required, as mentioned in the article, for the engineering assessment result, but for the clinical assessment, disclosure of assessment information to users is required rather than a specified numerical value of a tolerable level.

In the future, I think the two performance assessments will be conducted appropriately, and by feeding them back to product technology, higher reliability at user level can be achieved.

\section{Possibility of introduction of future verification by the Measurement Law \\ Question (Naoto Kobayashi)}

This is related to the question for Discussion 7, but ultimately, is it preferable to have all-product verification regulated by the measurement law for the ear thermometer? For this to be done, I think it is necessary for technological development to become matured fairly and start "to wilt", so to say, but when do you think that will happen? Or, to what level does the technology go for that?

\section{Answer (Juntaro Ishii)}

As mentioned in the article, verification is done to inspect the performance of measuring instrument according to law (Measurement Law) under the responsibility and authority of the government, and the measurement at a certain level or higher is maintained in Japan. Since it is a system with direct involvement of the government, user's confidence is high, but it may be a disincentive factor to product development and marketing for manufacturers. As mentioned in the previous section, product technology development to improve long-term stability as well as to improve reliability of clinical assessment is continued for the ear thermometer, so I think a shift to verification scheme with strong technological enforcement requires a period of about five years or more to study the trend of product technology. Moreover, new skin thermometer applying the principle of the ear thermometer is being developed, and technological consideration including such new thermometers will become an issue in the future.

This article discusses the consideration at the time when manufacture and sales of the ear thermometer grew rapidly in Japan from technological aspect. However in the future, if ear thermometers become a specified measuring instrument according to the Measurement Law, it will be necessary to consider not only the technological assessment, but also the economic effect on corporate activities by Japanese manufacturers, effect on the entry of overseas companies to Japanese market, and relationship with the Pharmaceutical Affairs Law (administered by Ministry of Health, Welfare and Labor). In the global flow toward free trade as exemplified by FTA, verification scheme under government large-scale auspice should be limited to absolutely necessary items to maintain safety of the citizens and reliability of trade, and creation of rules for maintaining traceability and conformity to international standards is strongly demanded internationally. Also, as mentioned in the previous Answer, clinical assessment including medical knowledge is necessary in addition to dissemination of measurement standard and establishment of engineering assessment method to obtain reliability at user level, so I think the important issue will be to consider more practical approach for both Measurement Law and Pharmaceutical Affairs Law. 